\section{Adaptación marginal de coronas de disilicato de litio obtenidas mediante técnicas de escaneo (CAD/CAM): análisis in vitro con microscopía confocal}

\author{
Marginal adaptation of lithium disilicate \\ crowns obtained by scanning techniques \\ (CAD/CAM): in vitro confocal microscopy \\ analysis
}

\section{Resumen}

Objetivo. El objetivo de este estudio fue evaluar la adaptación marginal de coronas de disilicato de litio obtenidas mediante técnicas de escaneo (CAD/CAM), antes y después de la cristalización, a través de análisis in vitro con microscopía confocal (MC). Métodos. Fueron confeccionadas 16 réplicas en poliuretano a partir de la pieza 1.4, de modelo typodont, tallada para corona total. Las réplicas fueron divididas en dos grupos, de acuerdo a la técnica de escaneo: Técnica Indirecta (Grupo IND, n=08), donde modelos de yeso fueron escaneados con escáner de laboratorio (inEos X5, Sirona Dental Systems) y Técnica Directa (Grupo DIR, n=08), donde modelos typodont fueron escaneados con escáner intraoral (CEREC BlueCam, Sirona Dental Systems). A seguir, se fresaron (inLab MC XL, Sirona Dental Systems) coronas en disilicato de litio (IPS e.max CAD, Ivoclar Vivadent) y se adaptaron a las réplicas. Se evaluó la adaptación marginal con análisis de $\mathrm{MC}$ en dos momentos, antes y después de la cristalización del disilicato de litio. Los datos fueron analizados con la prueba de Mann-Whitney, $t$ de Student y Wilcoxon $(\alpha=0,05)$. Resultados. Hubo una diferencia estadísticamente significativa en la adaptación marginal horizontal entre los grupos IND y DIR después de la cristalización $(\mathrm{p}=0,05)$. En el grupo IND, la comparación de la adaptación marginal vertical antes y después de la cristalización mostró una diferencia estadísticamente significativa $(\mathrm{p}=0,038)$. Conclusiones. Las coronas de disilicato de litio obtenidas mediante escaneo directo (CAD/CAM) presentaron menor desajuste marginal vertical. La etapa de cristalización afectó la adaptación marginal de las coronas.

Palabras clave: Cerámica; Adaptación marginal dental; Diseño asistido por computadora; Microscopía confocal (fuente: DeCS BIREME).

\begin{abstract}
Objective. This study aimed to evaluate lithium disilicate marginal adaption on crowns by scanning techniques (CAD/CAM), before and after crystallization, through confocal microscopy $(\mathrm{CM})$ in vitro analysis. Methods. Sixteen polyurethane replicas were performed from tooth 1.4, of a typodont model, prepared for a full crown. The replicas were divided into two groups, according to the scanning technique: Indirect Technique (Group IND, $\mathrm{n}=08$ ), where dental stone models were scanned with a laboratory scanner (inEos X5, Sirona Dental Systems) and Direct Technique (Group DIR, $\mathrm{n}=08$ ), where
\end{abstract}

Artículo Original

Bruna Neves de Freitas 1,a, Karen Pintado-Palomino 2,b, Bruna Santos Honório Tonin 1,a, Pedro Bastos Cruvinel 1,a, Ana Paula Macedo ${ }^{1, b}$, Camila Tirapelli ${ }^{1, b}$, Takami Hirono Hotta ${ }^{1,6}$, Wilson Matsumoto 1,6

${ }^{1}$ Universidade de São Paulo, Faculdade de Odontologia de Ribeirão Preto, Ribeirão Preto- São Paulo, Brasil.

${ }^{2}$ Ministerio de Salud, Cusco, Perú.

${ }^{a}$ Magíster en Ciencias.

${ }^{b}$ Doctor en Ciencias.

\section{Correspondencia:}

Bruna Neves de Freitas: bnevesfreitas@usp.br Av. Café s/n, Monte Alegre, 14040-904. Ribeirão PretoSão Paulo, Brasil.

ORCID: 0000-0001-7523-837X

\section{Coautores:}

Karen Pintado-Palomino: karpintado@gmail.com ORCID: 0000-0002-5677-3673

Bruna Santos Honório Tonin: bruna.tonin@usp.br ORCID: 0000-0001-7949-9980

Pedro Bastos Cruvinel: pedro.cruvinel@usp.br ORCID: 0000-0002-8323-355X Ana Paula Macedo: anapaula@forp.usp.br ORCID: 0000-0002-7716-106X Camila Tirapelli: catirapelli@forp.usp.br ORCID: 0000-0001-5020-6515

Takami Hirono Hotta: takamihotta@forp.usp.br ORCID: 0000-0003-1878-4504

Wilson Matsumoto: wmatsumoto@forp.usp.br ORCID: 0000-0002-5893-2850

\section{Editora:}

Rosse Mary Falcón-Antenucci

Universidad Inca Garcilaso de la Vega, Perú.

\section{Conflicto de intereses: los autores declaran no} tener conflictos de interés.

Fuente de financiamiento: Coordinación de Perfeccionamiento de Personal de Nivel Superior - Brasil (CAPES). Código de Financiamiento 001

\section{Recibido: 29/01/21}

Aceptado: 22/02/21

Publicado: 01/04/21 
typodont models were scanned with an intraoral scanner (CEREC BlueCam, Sirona Dental Systems). Then, the lithium disilicate crowns (IPS e.max CAD, Ivoclar Vivadent) were milled (inLab MC XL, Sirona Dental Systems) and adapted to the replicas. Marginal adaptation was evaluated with CM analysis before and after lithium disilicate crystallization. Data were analyzed with the Mann-Whitney, t test, and Wilconxon test $(\alpha=0.05)$. Results. There was a statistically significant difference in horizontal marginal adaptation between IND and DIR groups after crystallization ( $p=0.05)$. In IND group, the comparison of vertical marginal adaptation before and after crystallization showed a statistically significant difference $(\mathrm{p}=0.038)$. Conclusions. Lithium disilicate crowns obtained by direct scanning technique (CAD/CAD) showed less vertical marginal maladjustment. The crystallization stage affected the crown's marginal adaptation.

Keywords: Ceramics; Dental marginal adaptation; Computer aided design; Microscopy confocal (source: MeSH NLM).

\section{Introducción}

Actualmente, en la práctica clínica odontológica existe un aumento en la demanda de los pacientes por restauraciones protéticas de cerámicas libres de metal, debido a su naturalidad y propiedades estéticas, ya que evitan la corrosión, pigmentación gingival y reacciones alérgicas ${ }^{1,2}$.

De forma tradicional, las coronas dentales son confeccionadas a partir de impresiones convencionales con elastómeros. Sin embargo, los avances en la tecnología han permitido la introducción de otros métodos digitales como el sistema de diseño y fabricación asistido por computadora o computer-aided design and manufacturing (CAD/CAM), el cual reduce el número de procedimientos y mejora el confort del paciente. En este sistema existen varias técnicas para la captura de imágenes de las estructuras orales, que incluyen el escaneo directo del preparo dental o el escaneo indirecto de modelos de yeso. A partir de estas imágenes, la restauración es diseñada en un programa y confeccionada por una fresadora utilizando bloques cerámicos pre-fabricados ${ }^{3-5}$.

Independientemente del método utilizado para la obtención del registro del diente preparado, uno de los factores imprescindibles para el éxito clínico es una adecuada adaptación marginal de la corona al preparo dental. Una adaptación inadecuada conlleva a la solubilidad del cemento, microfiltración, acúmulo de biofilm ${ }^{6-8}$ y consecuentemente, afecta la longevidad y éxito del tratamiento. La adaptación marginal interna, que corresponde al espacio interno a ser ocupado por el cemento, necesita ser uniforme de forma que no comprometa la retención y/o reducción de la resistencia a la fractura de la restauración, principalmente cuando se trata de un material cerámico, debido a su mayor fragilidad en comparación a las aleaciones metálicas ${ }^{9}$.

En ese contexto, en el sistema CAD/CAM, las dimensiones del espacio interno de la interfaz diente/restauración es determinado a través de una herramienta espaciadora digital ${ }^{10}$. Sin embargo, estudios previos han reportado que las dimensiones determinadas en el proceso de CAD no corresponderían a los hallados después del proceso de fresado del material, afectando la adaptación marginal ${ }^{10-13}$. Así mismo, se ha reportado inconsistencia entre estudios que evaluaron el espacio marginal de restauraciones obtenidas por impresión convencional con silicona y escaneo indirecto del modelo. No en tanto, estas técnicas resultaron en restauraciones clínicamente aceptables ${ }^{1}$.

Por otro lado, sistemas modernos como el IPS e-max CAD está siendo utilizado ampliamente en la práctica clínica debido a su versatilidad. Se trata de un bloque de cerámica a base de disilicato de litio, empleado para la tecnología CAD/CAM, el cual debe someterse a un proceso de cristalización, conforme las instrucciones del fabricante. La cristalización es necesaria para que las propiedades finales del disilicato de litio sean alcanzadas ${ }^{14}$. Sin embargo, se ha reportado que el proceso de cristalización altera la relación corona-diente, reduciendo el espacio interno e impidiendo la adaptación adecuada de la restauración obtenida por la técnica de escaneo directa e indirecta ${ }^{15}$.

Por tanto, el objetivo de este estudio fue evaluar la adaptación marginal de coronas de disilicato de litio obtenidas mediante técnicas de escaneo directa e indirecta del sistema CAD/CAM, antes y después de la cristalización, a través de análisis in vitro con microscopía confocal.

\section{Métodos}

Diseño experimental. En este estudio in vitro, la variable evaluada fue la adaptación marginal, vertical y horizontal, con dos factores de variación: técnica de escaneo (directo e indirecto) y tiempo (antes y después de la cristalización). Se confeccionaron 16 réplicas en poliuretano de dientes artificiales preparados para corona total. Las 16 réplicas fueron distribuidas en dos grupos, de acuerdo a la técnica de escaneo a ser realizado: Escaneo Directo - Grupo IND ( $\mathrm{n}=08)$ y Escaneo Indirecto - Grupo DIR (n=08). El análisis de la adaptación marginal se realizó en dos tiempos, antes y después de la cristalización de las coronas de disilicato de litio.

Confección de muestras. Para la confección de muestras, inicialmente se realizó el tallado, para corona completa, en un diente artificial (pieza 1.4) de un modelo Typodont (Manequins Odontológicos Marília - Brasil), siguiendo los principios de preparación dental ${ }^{16}$. Se utilizó una fresa diamantada tronco-cónica de extremidad 
redondeada no 2136 (KG-Sorensen, Cotia, SP, Brasil) y se realizó la reducción de la superficie oclusal $(1,5 \mathrm{~mm})$, superficies axiales $(1,5 \mathrm{~mm})$ y una línea de terminación en chamfer con un ancho de $1,0 \mathrm{~mm}$. El acabado de la preparación se realizó con una punta diamantada de grano fino no 2136F (KG-Sorensen, Cotia, SP, Brasil) y con un instrumento manual de Black (SS White Duflex, Juiz de Fora, MG, Brasil).

A seguir, el diente preparado se duplicó con silicona (Silicone Master, Talmax, Curitiba, PR, Brasil) y posteriormente se obtuvo 16 réplicas en resina de poliuretano (F160, Axson, Socorro, São Paulo, Brasil). En estas réplicas se realizaron perforaciones estandarizadas, que sirvieron como puntos de referencia para la lectura de los análisis. Para ello, se trazó una línea por el centro de cada superficie del diente (labial, palatina, mesial y distal), a $1 \mathrm{~mm}$ del margen gingival, donde se perforó con una fresa de carburo de tungsteno $n^{\circ} 06$, posicionada en un paralelígrafo.

Las 16 réplicas fueron divididas en dos grupos experimentales, de acuerdo a la técnica de escaneo.

Técnica de escaneo. Incluyó:

Escaneo indirecto (Grupo IND=08): ocho réplicas fueron posicionadas en modelos Typodont, se siguió con la impresión completa del modelo con silicona de adición (Express XT, 3 M ESPE, Sumaré, São Paulo, Brasil) y posterior obtención de modelos en yeso tipo IV. Estos modelos fueron escaneados con escáner a laser de laboratorio (inEos X5; Sirona Dental Systems $\mathrm{GmbH}$, Bensheim, Hessen, Alemania).

A partir de las imágenes obtenidas del escaneo, un único operador diseñó una corona en el programa Sirona Connect (inLab SW, versión 15.1, Sirona Dental Systems $\mathrm{GmbH}$, Bensheim, Hessen, Alemania) con un espaciador radial de $40 \mu \mathrm{m}$ en toda la preparación dental, valor determinado en estudios previos ${ }^{13,17}$.

Una vez finalizado el diseño de la corona total, las imágenes fueron enviadas a la fresadora (inLab MC XL; Sirona Dental Systems GmbH Bensheim, Hessen, Alemania) para la confección de coronas a partir de bloques de cerámica de disilicato de litio (IPS e.max CAD, Ivoclar Vivadent, Schaan, Liechtenstein). El fresado se realizó en el estado parcialmente cristalizado del material. Las coronas no fueron glaseadas ni maquilladas.

Escaneo directo (Grupo DIR=08): ocho réplicas fueron posicionadas en modelos Typodont. Una vez posicionadas, se realizó una cobertura de dióxido de titanio (CEREC Optispray; Sirona Dental Systems GmbH Bensheim, Hessen, Germany). A seguir, se procedió al escaneo directo del diente preparado con el escáner BlueCam CEREC (Sirona Connect; Sirona Dental Systems). Las coronas fueron diseñadas y confeccionas siguiendo el mismo método del Grupo IND.

Análisis dimensional de la adaptación marginal. El análisis dimensional de la adaptación marginal se realizó en dos tiempos, antes y después de la cristalización de la cerámica de disilicato de litio. Las 16 coronas fueron cristalizadas en un horno específico EP 3000 G2 (Ivoclar, Vivadent Schaan, Liechtenstein) de $403^{\circ} \mathrm{C}$ a $840^{\circ} \mathrm{C}$ con $60 \%$ min siguiendo las instrucciones del fabricante.

La adaptación marginal vertical y horizontal fue evaluada por medio de un microscopio confocal a laser LEXT OLS 4000 (Olympus, Japón). Para estandarizar la posición del conjunto réplica dental - coronas adaptadas en el microscopio, se confeccionaron cuatro soportes en silicona de condensación (Zetalabor, Zhermack, Badia Polesine RO, Italia), uno para cada superficie dental (vestibular, palatina, mesial y distal). Se obtuvieron cuatro imágenes en $3 \mathrm{D}$ de alta resolución (5x y 108x) por cada muestra. A seguir, se mensuró la adaptación vertical y horizontal a partir de la referencia hecha en las réplicas en poliuretano. Posteriormente, se obtuvo tres medidas por cada imagen, una en dirección del centro de la referencia de la superficie respectiva y otras dos en dirección tangente al margen derecho e izquierdo de la referencia.

Análisis estadístico. Inicialmente, se realizó una prueba de normalidad (Shapiro-Wilk) para determinar la distribución de los datos. Para la comparación entre los grupos IND y DIR, se utilizó la prueba de Mann-Whitney y t de Student. Para la comparación de los resultados antes y después de la cristalización, se utilizó la prueba de Wilconxon y t de Student. Se determinó un nivel de significancia del 95\% ( $\leq \leq 0,05)$. El análisis estadístico se realizó a través del programa SPSS para Windows, versión 20.0. (IBM Corp. Armonk, New York).

\section{Resultados}

Los resultados obtenidos en la prueba de normalidad son presentados en la Tabla 1. A continuación, la Tabla 2 muestra la estadística descriptiva de los datos analizados, donde se observa valores de espacio marginal vertical mayores después del proceso de cristalización en el grupo IND y DIR.

Los resultados de la comparación entre los grupos IND y DIR mostraron que hubo una diferencia estadísticamente significativa $(\mathrm{p}<0,05)$ en la adaptación marginal horizontal después de la cristalización, donde el grupo IND presentó valores significativamente menores que el grupo DIR (Tabla 3).

Por otra parte, en la comparación de los tiempos de evaluación (antes y después de la cristalización de la cerámica) se observó que en el grupo IND hubo una diferencia estadísticamente significativa $(p=0,038)$ en la adaptación marginal vertical, donde los valores después de la cristalización fueron significativamente mayores. En el grupo DIR, esta diferencia fue observada en la adaptación marginal horizontal, mostrando valores significativamente mayores después de la cristalización $(\mathrm{p}=0,005)$ (Tabla 4).

\section{Discusión}

Los resultados mostraron que la técnica de escaneo y la etapa de la cristalización influyeron en la adaptación de la restauración de cerámica de disilicato de litio. En 
Tabla 1. Prueba de normalidad

\begin{tabular}{|c|c|c|c|c|c|}
\hline \multirow{2}{*}{ Método } & \multirow{2}{*}{ Variable } & \multirow{2}{*}{ Grupo } & \multicolumn{3}{|c|}{ Shapiro-Wilk } \\
\hline & & & Estadística & $\mathbf{n}$ & Sig. \\
\hline \multirow{8}{*}{ Microscopía Confocal } & \multirow{2}{*}{$\begin{array}{l}\text { Adaptación Marginal Vertical } \\
\text { Antes }\end{array}$} & IND & 0.903 & 8 & 0.306 \\
\hline & & DIR & 0.827 & 8 & 0.055 \\
\hline & \multirow{2}{*}{$\begin{array}{l}\text { Adaptación Marginal Vertical } \\
\text { Después }\end{array}$} & IND & 0.885 & 8 & 0.210 \\
\hline & & DIR & 0.790 & 8 & $0.023^{*}$ \\
\hline & \multirow{2}{*}{$\begin{array}{l}\text { Adaptación Marginal Horizontal } \\
\text { Antes }\end{array}$} & IND & 0.856 & 8 & 0.110 \\
\hline & & DIR & 0.778 & 8 & $0.017^{*}$ \\
\hline & \multirow{2}{*}{$\begin{array}{l}\text { Adaptación Marginal Horizontal } \\
\text { Después }\end{array}$} & IND & 0.937 & 8 & 0.586 \\
\hline & & DIR & 0.803 & 8 & $0.031^{*}$ \\
\hline
\end{tabular}

*Distribución no normal

Tabla 2. Medidas de posición central, de dispersión de las medidas en relación, a los grupos y tiempo de evaluación.

\begin{tabular}{|c|c|c|c|c|c|c|c|c|c|}
\hline Variable & Grupo & Tiempo & Media & $\begin{array}{c}\text { Desviación } \\
\text { estándar }\end{array}$ & Mediana & Q1 & Q3 & $\min$ & máx. \\
\hline \multirow{4}{*}{$\begin{array}{c}\text { Marginal } \\
\text { Vertical }\end{array}$} & \multirow{2}{*}{ IND } & Antes & 181,62 & 84,33 & 152,56 & 129,61 & 231,42 & 81,16 & 353,01 \\
\hline & & Después & 215,93 & 81,29 & 200,72 & 144,73 & 284,14 & 139,13 & 363,09 \\
\hline & \multirow{2}{*}{ DIR } & Antes & 196,80 & 48,86 & 189,52 & 171,69 & 202,95 & 136,89 & 305,23 \\
\hline & & Después & 279,57 & 172,55 & 219,56 & 177,76 & 353,10 & 128,30 & 662,07 \\
\hline \multirow{4}{*}{$\begin{array}{l}\text { Marginal } \\
\text { Horizontal }\end{array}$} & \multirow{2}{*}{ IND } & Antes & 102,89 & 48,91 & 87,97 & 63,46 & 149,07 & 56,83 & 189,90 \\
\hline & & Después & 77,73 & 35,06 & 66,70 & 51,49 & 103,83 & 35,13 & 143,43 \\
\hline & \multirow{2}{*}{ DIR } & Antes & 80,75 & 56,06 & 60,02 & 51,29 & 111,98 & 28,86 & 200,58 \\
\hline & & Después & 165,24 & 127,64 & 102,94 & 74,60 & 249,93 & 65,84 & 428,25 \\
\hline
\end{tabular}

Tabla 3. Comparación de la adaptación marginal vertical y horizontal $(\mu \mathrm{m})$ entre los grupos analizados por microscopía confocal a laser, antes y después de la cristalización

\begin{tabular}{ccc}
\hline Región & Grupo IND vs Grupo DIR & $\mathbf{p}^{*}$ \\
\hline Marginal Vertical & Antes & 0,505 \\
& Después & 0,574 \\
\hline \multirow{2}{*}{ Marginal Horizontal } & Antes & 0,195 \\
& Después & $0,055^{* *}$ \\
\hline
\end{tabular}

*U de Mann-Whitney

** diferencia estadísticamente significante $(p \leq 0,05)$

Tabla 4. Comparación entre los tiempos de evaluación para la adaptación marginal vertical y horizontal $(\mu \mathrm{m})$ para los diferentes grupos experimentales

\begin{tabular}{ccc}
\hline Región & Antes vs Después & $\mathrm{p}^{*}$ \\
\hline \multirow{2}{*}{ Adaptación Marginal Vertical } & Grupo IND & $0,038^{\mathrm{r}, *}$ \\
& Grupo DIR & $0,241^{\text {, }}$ \\
\multirow{2}{*}{ Adaptación Marginal Horizontal } & Grupo IND & $0,076^{\mathrm{r}}$ \\
& Grupo DIR & $0,005^{\mathrm{*}, *}$ \\
\hline
\end{tabular}

r prueba t de Student; ${ }^{\vee}$ prueba de Wilcoxon; * diferencia estadísticamente significativa $(p \leq 0,05)$,

ambos grupos, se encontró una diferencia en la adaptación marginal después de la cristalización de la cerámica, evidenciando una alteración en la adaptación horizontal en el Grupo DIR (antes=60,02 vs después $=102,94$, $\mathrm{p}=0,005)$ y vertical en el grupo IND (antes $=181,62$ vs después $=215,93, \mathrm{p}=0,038)$. El aumento de los valores encontrados después de la cristalización indica una mayor desadaptación marginal de la restauración cerámica a la preparación dental. Así mismo, los resultados del análisis con microscopía confocal a laser mostraron que hubo diferencia significativa en la adaptación marginal horizontal entre los grupos, siendo que el grupo IND presentó valores significativamente menores después de la cristalización. 
La adaptación final de las coronas completas de disilicato de litio obtenidas por impresión convencional y digital vienen siendo analizadas en diversos estudios ${ }^{18-22}$. Sin embargo, poca información existe sobre el efecto de la cristalización en la adaptación del disilicato de litio ${ }^{23}$.

El aumento de la distancia marginal entre la restauración y el margen dental después de la cristalización del disilicato de litio sugiere que ocurrió una contracción, la cual también fue observada por Kim; Oh y Uhm ${ }^{23}$, en la que hubo una reducción de la desadaptación axial de 45,23 $\mu \mathrm{m}$ para $24,74 \mu \mathrm{m}$. La desviación estándar encontrada resultó en la diferencia estadísticamente significativa, pese a que se puede observar en la tabla 2 un valor medio mayor para el grupo DIR.

La observación de todos los factores que pueden influenciar en la adaptación de la restauración indirecta es relevante. Sin embargo, es necesario enfatizar la importancia de los valores de la adaptación marginal vertical. Este es un local crítico por ser menos propenso a corrección en el ajuste manual ${ }^{24,25}$. La distancia vertical es ocupada apenas por el agente de cementación, cuyo exceso puede aumentar la desadaptación vertical ${ }^{26}$ estando sujeto a la disolución en el medio bucal ${ }^{27}$. En este sentido, el grupo DIR, que corresponde a la técnica de escaneo directo resultó en menor desadaptación marginal. Es probable que la inclusión de etapas convencionales del grupo IND (toma de impresión y vaciado en yeso), pueda ser un factor de inclusión de discrepancia favoreciendo la desadaptación marginal de la restauración final.

Por otra parte, los valores de adaptación marginal vertical antes y después de la cristalización del disilicato de litio, por ambos métodos de escaneo fueron superiores al sugerido por McLean y Fraunhofer ${ }^{28}$ - inferior a 120 $\mu \mathrm{m}$-, utilizado como parámetro en diversos estudios 19, 21, 23 y considerado como límite de una adaptación clínicamente aceptable ${ }^{20}$. Los valores obtenidos en este estudio (superiores a $120 \mu \mathrm{m}$ ) pueden ser justificados por factores inherentes a las técnicas de la obtención y fabricación de la restauración afectando la adaptación interna y marginal de la prótesis ${ }^{10}$.

En la práctica clínica, un posible contacto proximal fuerte es eliminado con ajustes en el momento de la cementación. Este procedimiento después del fresado es un factor que puede afectar los valores de adaptación de la restauración y podría explicar los valores por encima del límite considerado de $120 \mu \mathrm{m}$. El ajuste previo de la pieza visando el mejor asentamiento no fue realizado debido a la dificultad en la estandarización. Los resultados de este estudio, sugieren que la etapa de ajuste de laboratorio y, si es necesario clínico, puede reducir la distancia marginal entre el diente y la restauración protética.

Así mismo, el espacio interno determinado por la etapa CAD también influye en la adaptación marginal ${ }^{10}$. En este estudio se estableció un espacio interno de $40 \mu \mathrm{m}$, similar al utilizado por Rajan et al. ${ }^{13}$. No en tanto, este valor no está bien establecido en la literatura, variando entre $0 \mu \mathrm{m}^{29}, 20 \mu \mathrm{m}^{21,30}, 50 \mu \mathrm{m}^{19}$ y $60 \mu \mathrm{m}^{31}$. Kim, Oh y Uhm ${ }^{23}$ sugieren que el valor de la configuración del espacio interno determinado en la etapa CAD considere la reducción de éste después de la cristalización del material. Por su parte, un espacio reducido impide el asentamiento marginal llevando a un aumento en el desajuste marginal vertical en razón del contacto prematuro entre la pared axial y la corona de disilicato de litio.

Adicionalmente, la configuración del preparo dental y de su línea de terminación son factores que pueden influir en la adaptación de la pieza al diente pilar ${ }^{6,32}$ debido a la digitalización por el escáner y, consecuentemente, el diseño de la restauración en la terminación cervical o por la definición en la visualización del microscopio confocal a laser. Siendo así, la terminación utilizada en este estudio está de acuerdo con lo indicado para el escaneo de preparo, que requiere una terminación suave debido a la limitación del escáner al copiar ángulos agudos ${ }^{32-34}$. Sin embargo, esta característica vista en el microscopio confocal puede ser desventajosa comparada a un término de márgenes definidas. Las técnicas de visualización del espacio interno utilizando la microtomografía computarizada y análisis cuantitativo volumétrico por medio de softwares pueden favorecer el desarrollo de los resultados en este campo científico ${ }^{15,35-37}$.

A pesar que los valores de adaptación marginal sean clínicamente aceptables entre los métodos convencionales y digitales, es necesario el mejoramiento de estos sistemas digitales con el objetivo que sean superiores a las técnicas convencionales y justifique la inversión en equipos y adiestramiento al cirujano dentista y técnico en prótesis dental ${ }^{10,11,30}$.

Se sugiere que diferentes valores de adaptación sean abordados en futuras investigaciones, bien como diferentes tecnologías de fabricación y metodologías que permitan la cuantificación del volumen interno entre diente/restauración. Finalmente, de acuerdo con los resultados y las limitaciones de este estudio, se concluyó que las coronas de disilicato de litio obtenidas mediante la técnica de escaneo directo, del sistema CAD/CAM, presentaron menor desajuste marginal vertical y que la etapa de cristalización afectó la adaptación marginal de las coronas.

\section{Referencias bibliográficas}

1. Memari Y, Mohajerfar M, Armin A, Kamalian F, Rezayani V, Beyabanaki E. Marginal adaptation of CAD/ CAM all-ceramic crowns made by different impression methods: a literature review. J Prosthodont. 2019;28(2):536-44. DOI: 10.1111/jopr.12800.

2. Fradeani M, Redemagni M. An 11-year clinical evaluation of leucite reinforced glass-ceramic crowns: a retrospective study. Quintessence Int [Internet]. 2002 [citado el 28 de enero de 2021]; 33(7):503-10. Disponible en: https://www.researchgate.net/publication/11220867_ An_11-Year_Clinical_Evaluation_of_Leucite-Reinforced_Glass-Ceramic_Crowns_A_Retrospective_Study\#fullTextFileContent.

3. Davidowitz G, Kotick PG. The use of CAD/CAM in dentistry. Dent Clin North Am. 2011;55(3):559-70. DOI: 10.1016/j.cden.2011.02.011. 
4. Dehailan LA. Review of the current status of all-ceramic restorations. [Internet] 2009. Disponible en: http://www.iusd.iupui.edu/index.php/download_file/ view/418/488/.

5. Santos MJ, Costa MD, Rubo JH, Pegoraro LF, Santos GC Jr. Current all-ceramic systems in dentistry: a review. Compend Contin Educ Dent [Internet]. 2015 (citado el 28 de enero de 2021]; 36(1):31-7, quiz 38,40. Disponible en: https://www.researchgate.net/publication/274262885_Current_All-Ceramic_Systems_in_ Dentistry_A_Review.

6. Contrepois M, Soenen A, Bartala M, Laviole O. Marginal adaptation of ceramic crowns: a systematic review. J Prosthet Dent. 2013;110(6):447-54. DOI: 10.1016/j. prosdent.2013.08.003.

7. Orstavik D, Orstavik J. In vitro attachment of Streptococcus sanguis to dental crown and bridge cements. J Oral Rehabil. 1976;3(2):139-44. DOI: 10.1111/j.13652842.1976.tb00937.x

8. Yüksel E, Zaimoglu A. Influence of marginal fit and cement types on microleakage of all-ceramic crown systems. Braz Oral Res. 2011;25(3):261-66. DOI: 10.1590/ s1806-83242011000300012.

9. Akın A, Toksavul S, Toman M. Clinical Marginal and Internal Adaptation of Maxillary Anterior Single All-Ceramic Crowns and 2-year Randomized Controlled Clinical Trial. J Prosthodont. 2015;24(5):345-50. DOI: 10.1111/jopr.12217.

10. Mously HA, Finkelman M, Zandparsa R, Hirayama H. Marginal and internal adaptation of ceramic crown restorations fabricated with CAD/CAM technology and the heat-press technique. J Prosthet Dent. 2014;112(2):24956. DOI: $10.1016 /$ j.prosdent.2014.03.017.

11. Anadioti E, Aquilino SA, Gratton DG, et al. 3D and 2D marginal fit of pressed and CAD/CAM lithium disilicate crowns made from digital and conventional impressions. J Prosthodont. 2014;23(8):610-7. DOI: 10.1111/ jopr.12180.

12. Gold SA, Ferracane JL, da Costa J. Effect of crystallization firing on marginal gap of CAD/CAM fabricated Lithium Disilicate crowns. J Prosthodont. 2018;27(1):636. DOI: $10.1111 /$ jopr. 12638 .

13. Rajan BN, Jayaraman S, Kandhasamy B, Rajakumaran I. Evaluation of marginal fit and internal adaptation of zirconia copings fabricated by two CAD - CAM systems: an in vitro study. J Indian Prosthodont Soc. 2015;15(2):173-8. DOI: 10.4103/0972-4052.159970.

14. Giordano R, McLaren EA. Ceramics overview: classification by microstructure and processing methods. Compend Contin Educ Dent [Internet]. 2010 (citado el 28 de enero de 2021]; 31(9):682-4, 686, 688 passim; quiz 698, 700. Disponible en: http://www.moderndentistrymedia.com/may_june2014/mclaren.pdf.

15. De Freitas BN, Tonin BSH, Macedo AP, Dos Santos TMP, De Mattos MDGC, Hotta TH, et al. Adaptation accuracy of milled lithium disilicate crowns: A $2 \mathrm{D}$ and $3 \mathrm{D}$ microCT analysis. J Esthet Restor Dent. 2020;32(4):403-9. DOI: 10.1111/jerd.12574.

16. Goodacre CJ, Campagni WV, Aquilino SA. Tooth preparations for complete crowns: an art form based on scientific principles. J Prosthet Dent. 2001;85(4):36376. DOI: $10.1067 / \mathrm{mpr} .2001 .114685$.

17. Nakamura T, Dei N, Kojima T, Wakabayashi K. Marginal and internal fit of Cerec 3 CAD/CAM all-ceramic crowns. Int J Prosthodont. 2003;16(3):244-8. DOI: 10.1067/mpr.2001.114685.

18. Abdel-Azim T, Rogers K, Elathamna E, Zandinejad A, Metz M, Morton D. Comparison of the marginal fit of lithium disilicate crowns fabricated with CAD/CAM technology by using conventional impressions and two intraoral digital scanners. J Prosthet Dent. 2015;114(4):554-9. DOI: 10.1016/j.prosdent.2015.04.001.

19. Ahrberg D, Lauer HC, Ahrberg M, Weigl P. Evaluation of fit and efficiency of CAD/CAM fabricated all-ceramic restorations based on direct and indirect digitalization: a double-blinded, randomized clinical trial. Clin Oral Investig. 2016;20(2):291-300. DOI: 10.1007/s00784-015-1504-6.

20. Mounajjed R, M Layton D, Azar B. The marginal fit of E.max Press and E.max CAD lithium disilicate restorations: A critical review. Dent Mater J. 2016;35(6):83544. DOI: $10.4012 / \mathrm{dmj} .2016-008$.

21. Mostafa NZ, Ruse ND, Ford NL, Carvalho RM, Wyatt CCL. Marginal Fit of Lithium Disilicate Crowns Fabricated Using Conventional and Digital Methodology: A Three-Dimensional Analysis. J Prosthodont. 2018;27(2):145-52. DOI: 10.1111/jopr.12656.

22. Pradíes G, Zarauz C, Valverde A, Ferreiroa A, Martínez-Rus F. Clinical evaluation comparing the fit of all-ceramic crowns obtained from silicone and digital intraoral impressions based on wavefront sampling technology. J Dent. 2015;43(2):201-8. DOI: 10.1016/j. jdent.2014.12.007.

23. Kim JH, Oh S, Uhm SH. Effect of the Crystallization Process on the Marginal and Internal Gaps of Lithium Disilicate CAD/CAM Crowns. Biomed Res Int. 2016;2016:8635483. DOI: 10.1155/2016/8635483.

24. Holmes JR, Bayne SC, Holland GA, Sulik WD. Considerations in measurement of marginal fit. J Prosthet Dent. 1989;62(4):405-8. DOI: 10.1016/00223913(89)90170-4.

25. Ng J, Ruse D, Wyatt C. A comparison of the marginal fit of crowns fabricated with digital and conventional methods. J Prosthet Dent. 2014;112(3):555-60. DOI: 10.1016/j.prosdent.2013.12.002.

26. Beschnidt SM, Strub JR. Evaluation of the marginal accuracy of different all-ceramic crown systems after simulation in the artificial mouth. J Oral Rehabil. 1999;26(7):582-93. DOI: 10.1046/j.13652842.1999.00449.x.

27. Jacobs MS, Windeler AS. An investigation of dental luting cement solubility as a function of the marginal gap. J Prosthet Dent. 1991;65(3):436-42. DOI: 10.1016/0022-3913(91)90239-s.

28. McLean JW, von Fraunhofer JA. The estimation of cement film thickness by an in vivo technique. Br Dent J. 1971;131(3):107-11. DOI: 10.1038/sj.bdj.4802708.

29. Neves FD, Prado CJ, Prudente MS, Carneiro TA, Zancopé $\mathrm{K}$, Davi LR, et al. Micro-computed tomography evaluation of marginal fit of lithium disilicate crowns fabricated 
by using chairside CAD/CAM systems or the heat-pressing technique. J Prosthet Dent. 2014;112(5):1134-40. DOI: $10.1016 /$ j.prosdent.2014.04.028.

30. Alfaro DP, Ruse ND, Carvalho RM, Wyatt CC. Assessment of the Internal Fit of Lithium Disilicate Crowns Using Micro-CT. J Prosthodont. 2015;24(5):381-6. DOI: 10.1111 /jopr.12274.

31. Salamoni Sinhori B, de Andrada MAC, Carpena Lopes G, Monteiro Junior S, Baratieri LN. Influence of teeth preparation finishing on the adaptation of lithium disilicate crowns. Int J Biomater. 2017;2017:2078526. DOI: $10.1155 / 2017 / 2078526$

32. Renne W, Wolf B, Kessler R, McPherson K, Mennito AS. Evaluation of the Marginal Fit of CAD/CAM Crowns Fabricated Using Two Different Chairside CAD/CAM Systems on Preparations of Varying Quality. J Esthet Restor Dent. 2015;27(4):194-202. DOI: 10.1111/ jerd.12148.

33. Beuer F, Schweiger J, Edelhoff D. Digital dentistry: an overview of recent developments for CAD/CAM generated restorations. Br Dent J. 2008;204(9):505-11. DOI: 10.1038/sj.bdj.2008.350.
34. Güth JF, Wallbach J, Stimmelmayr M, Gernet W, Beuer F, Edelhoff D. Computer-aided evaluation of preparations for CAD/CAM-fabricated all-ceramic crowns. Clin Oral Investig. 2013;17(5):1389-95. DOI: $10.1007 /$ s00784-012-0812-3.

35. Oğuz Eİ, Kılıçarslan MA, Özcan M, Ocak M, Bilecenoğlu B, Orhan K. Evaluation of denture base adaptation fabricated using conventional, subtractive and additive technologies: a volumetric micro-computed tomography analysis. J Prosthodont. 2021 Jan 7. DOI: 10.1111/jopr.13326. Online ahead of print.

36. Tasaka A, Okano H, Odaka K, Matsunaga S, Goto TK, Abe $S$, et al. Comparison of artificial tooth position in dentures fabricated by heat-curing and additive manufacturing. Aust Dent J. 2021 Jan 7. DOI: 10.1111/ adj.12817. Online ahead of print.

37. Bayrak A, Akat B, Ocak M, Kılıçarslan MA, Özcan M. Micro-Computed Tomography Analysis of Fit of Ceramic Inlays Produced with Different CAD Software Programs. Eur J Prosthodont Restor Dent. 2020 Dec 7. DOI: 10.1922/EJPRD_2046Bayrak06. Online ahead of print. 